\title{
The Solutions of Second-Order Linear Matrix Equations on Time Scales
}

\author{
Kefeng Li and Chao Zhang \\ School of Mathematical Sciences, University of Jinan, Jinan, Shandong 250022, China \\ Correspondence should be addressed to Chao Zhang; ss_zhangc@ujn.edu.cn
}

Received 11 July 2013; Accepted 29 August 2013

Academic Editor: Shurong Sun

Copyright (c) $2013 \mathrm{~K}$. Li and C. Zhang. This is an open access article distributed under the Creative Commons Attribution License, which permits unrestricted use, distribution, and reproduction in any medium, provided the original work is properly cited.

\begin{abstract}
This paper studies the solutions of second-order linear matrix equations on time scales. Firstly, the necessary and sufficient conditions for the existence of a solution of characteristic equation are introduced; then two diverse solutions of characteristic equation are applied to express general solution of the matrix equations on time scales.
\end{abstract}

\section{Introduction}

In this paper, we consider the solutions for the following second-order linear matrix equations:

$$
\left[P(t) X^{\Delta}(t)\right]^{\Delta}+Q X^{\Delta}(t)+R(t) X^{\sigma}(t)=0, \quad t \in \mathbb{T},
$$

where $P(t)=P \mu(t), R(t)=R / \mu(t), P, Q, R, X(t) \in \mathbf{R}^{\mathbf{m} \times \mathbf{m}}$, $X^{\Delta}$ is the delta derivative, $\sigma(t)$ is the forward jump operators, $X^{\sigma}(t):=X(\sigma(t)), \mu(t)$ is the graininess function, and $\mathbb{T}$ is an infinite isolated time scale, which is given by

$$
\mathbb{T}:=\left\{t_{0}, t_{1}, t_{2}, \ldots, t_{n}, \ldots\right\} \text {. }
$$

As a tool for establishing a unified framework for continuous and discrete analysis, a theory of dynamic equations on measure chains was introduced by Hilger in his Ph.D. thesis [1] in 1988. In many cases, it is necessary to study a special case of measure chains-time scales. In the last decade the investigation of dynamic systems on time scales has involved much interesting, including quite a few fields, such as the theory of calculus, the oscillation of the dynamic systems, the eigenvalue problems and boundary value problems, and partial differential equations on time scales, and so forth [25]. Up to now, there are few results about matrix equations on time scales. In 1998, Agarwal and Bohner [6] studied the quadratic functionals for second order matrix equations on time scales; in 2002, Erbe and Peterson [7] obtained oscillation criteria for a second-order self-adjoint matrix differential equation on time scales in terms of the eigenvalues of the coefficient matrices and the graininess function. The theory of dynamic systems on time scales is of very important theoretical significance and has a wide range of applications.

Based on the related literatures, the researches of solutions of matrix difference or differential equations have few results. In 1999, Barkatou [8] proposed an algorithm for the rational solutions of special matrix difference equations and discussed their applications; in 2003, Freiling and Hochhaus [9] investigated some properties of the solution for rational matrix difference equations; in 2004, Xu and Zhang [10] studied the representation of general solution for second order homogeneous matrix difference equations; in 2011, $\mathrm{Wu}$ and Zhou [11] obtained the particular solutions of one kind of second order matrix differential equations. Since the continuous case and the discrete case are two special cases of time scales, so, we study the solutions of second order linear matrix equations on time scales.

This paper is organized as follows. Section 2 introduces some basic concepts and fundamental theory about time scales. By using the characteristic equation of the matrix equation the solutions of (1) are obtained, which will be given in Section 3.

\section{Preliminaries}

In this section, some basic concepts and some fundamental results on time scales are introduced. 
Let $\mathbb{T} \subset \mathbf{R}$ be a nonempty closed subset. Define the forward and backward jump operators $\sigma, \rho: \mathbb{T} \rightarrow \mathbb{\mathbb { V }}$ by

$$
\sigma(t)=\inf \{s \in \mathbb{T}: s>t\}, \quad \rho(t)=\sup \{s \in \mathbb{T}: s<t\},
$$

where inf $\emptyset=\sup \mathbb{T}$, sup $\emptyset=\inf \mathbb{T}$. We put $\mathbb{T}^{k}=\mathbb{T}$ if $\mathbb{T}$ is unbounded above and $\mathbb{T}^{k}=\mathbb{T} \backslash(\rho(\max \mathbb{\mathbb { V }})$, $\max \mathbb{\mathbb { T }}]$ otherwise. The graininess functions $\nu, \mu: \mathbb{T} \rightarrow[0, \infty)$ are defined by

$$
\mu(t)=\sigma(t)-t, \quad v(t)=t-\rho(t)
$$

Let $f$ be a function defined on $\mathbb{T}$. $f$ is said to be (delta) differentiable at $t \in \mathbb{T}^{k}$ provided that there exists a constant $a$ such that, for any $\varepsilon>0$, there is a neighborhood $U$ of $t$ (i.e., $U=(t-\delta, t+\delta) \cap \mathbb{\mathbb { f }}$ for some $\delta>0)$ with

$$
|f(\sigma(t))-f(s)-a(\sigma(t)-s)| \leq \varepsilon|\sigma(t)-s|, \quad \forall s \in U
$$

In this case, denote $f^{\Delta}(t):=a$. If $f$ is (delta) differentiable for every $t \in \mathbb{T}^{k}$, then $f$ is said to be (delta) differentiable on $\mathbb{T}$. If $f$ is differentiable at $t \in \mathbb{T}^{k}$, then

$$
f^{\Delta}(t)= \begin{cases}\lim _{\substack{s \rightarrow t \\ s \in \mathbb{T}}} \frac{f(t)-f(s)}{t-s}, & \text { if } \mu(t)=0, \\ \frac{f(\sigma(t))-f(t)}{\mu(t)}, & \text { if } \mu(t)>0 .\end{cases}
$$

For convenience, we introduce the following results ([3, Lemma 1] and [4, Chapter 1]), which are useful in this paper.

Lemma 1. Let $f, g: \mathbb{T} \rightarrow \mathbf{R}$ and $t \in \mathbb{T}^{k}$. If $f$ and $g$ are differentiable at $t$, then $\mathrm{fg}$ is differentiable at $t$ and

$$
\begin{aligned}
(f g)^{\Delta}(t) & =f^{\sigma}(t) g^{\Delta}(t)+f^{\Delta}(t) g(t) \\
& =f^{\Delta}(t) g^{\sigma}(t)+f(t) g^{\Delta}(t) .
\end{aligned}
$$

\section{Main Results}

We assume throughout this paper that $P$ is an invertible matrix. It follows from Lemma $1,(6)$, and (2) that the matrix equation (1) can be written in the form

$$
X^{\sigma^{2}}(t)-B X^{\sigma}(t)-A X(t)=0, \quad t \in \mathbb{T},
$$

where $X^{\sigma^{2}}(t)=X^{\sigma}(\sigma(t)), B=2 E_{m}-P^{-1} Q-P^{-1} R, A=$ $P^{-1} Q-E_{m}$, and $E_{m}$ is the identity matrix.
Definition 2. The equation

$$
F(\Lambda)=\Lambda^{2}-B \Lambda-A=0,
$$

where $\Lambda \in \mathbf{R}^{\mathbf{m} \times \mathbf{m}}$, is called the characteristic equation of (8).

Definition 3. The functions

$$
F(\lambda)=\lambda^{2}-B \lambda-A, \quad h(\lambda)=\operatorname{det} F(\lambda)
$$

are called the eigenmatrix and eigenpolynomial of (8). Such a $\lambda$ which satisfies $h(\lambda)=0$ is called an eigenvalue of $F(\lambda)$.

In order to study the solutions of matrix equation (8), we now introduce some results about the characteristic equation (9).

Theorem 4. If $A B=B A$ and there exists $C \in \mathbf{R}^{\mathbf{m} \times \mathbf{m}}$ such that $C^{2}=B^{2}+4 A, B C=C B$, then the characteristic equation (9) has solutions.

Proof. Let $\Lambda_{1}=(1 / 2)(B+C), \Lambda_{2}=(1 / 2)(B-C)$. Then $\Lambda_{1}$ and $\Lambda_{2}$ are solutions of (9).

Remark 5. Generally, if $A B=B A$ and $C^{2}=B^{2}+4 A$, we cannot easily get $B C=C B$.

Example 6. Let

$$
A=\left(\begin{array}{cc}
\frac{1}{2} & 0 \\
\frac{1}{4} & \frac{1}{4}
\end{array}\right), \quad B=\left(\begin{array}{cc}
1 & 0 \\
2 & -1
\end{array}\right)
$$

Then

$$
\begin{gathered}
C=\left(\begin{array}{cc}
\sqrt{3} & 0 \\
\frac{1}{1+\sqrt{3}} & 1
\end{array}\right), \quad B C=\left(\begin{array}{cc}
\sqrt{3} & 0 \\
\frac{5+2 \sqrt{3}}{1+\sqrt{3}} & -1
\end{array}\right), \\
C B=\left(\begin{array}{cc}
\sqrt{3} & 0 \\
\frac{3+2 \sqrt{3}}{1+\sqrt{3}} & -1
\end{array}\right) .
\end{gathered}
$$

Obviously, $B C \neq C B$.

So, Huang and Chen [12] and J. Huang and H. Huang [13] obtained the following results.

Definition 7. Let $\lambda_{0}$ be an eigenvalue of $F(\lambda)$. Then

$$
V_{\lambda_{0}}=\left\{x \mid\left(\lambda_{0}^{2}-B \lambda_{0}-A\right) x=0, x \in \mathbf{R}^{\mathbf{m} \times \mathbf{1}}\right\}
$$

is called a characteristic subspace of $F(\lambda)$ corresponding to $\lambda_{0}$; the nonzero vector $x$ of $V_{\lambda_{0}}$ is called the eigenvector corresponding to $\lambda_{0}$.

Theorem 8. There exist the diagonalizable solutions of characteristic equation (9) if and only if the dimension of the sum of characteristic subspaces $V_{\lambda_{i}}$ is $n$; that is, $\operatorname{dim}\left\{\sum_{i=1}^{h} V_{\lambda_{i}}\right\}=m$, where $\lambda_{i}(i=1,2, \ldots, h)$ are the distinct eigenvalues of $F(\lambda)$. 
Definition 9. Let $\lambda_{i}, i=1,2, \ldots, s$, be eigenvalues of $F(\lambda)$. The extension vectors set of $V_{\lambda_{i}}$ are as follows:

$$
\begin{aligned}
E V_{\lambda_{i}}=\{( & \left.x_{0}, x_{1}, \ldots, x_{k}\right) \mid \\
& \left(\lambda_{i}^{2}-B \lambda_{i}-A\right) x_{0}=0, \\
& \left(\lambda_{i}^{2}-B \lambda_{i}-A\right) x_{1} \\
& =-\left(2 \lambda_{i} E_{m}-B\right) x_{0}, \quad k \leq k_{i}-1, \\
& \left(\lambda_{i}^{2}-B \lambda_{i}-A\right) x_{k} \\
& \left.=-\left(2 \lambda_{i} E_{m}-B\right) x_{k-1}-x_{k-2} E_{m}\right\},
\end{aligned}
$$

where $k_{i}$ is the multiplicity of $\lambda_{i},\left(x_{0}, x_{1}, \ldots, x_{k}\right)$ is called the component of $E V_{\lambda_{i}}$.

Theorem 10. The characteristic equation (9) has solutions if and only if there exist some components $\left(x_{0}, x_{1}, \ldots\right.$ ,$\left.x_{k}\right), \ldots,\left(y_{0}, y_{1}, \ldots, y_{q}\right)$ of the set $\sum_{i=1}^{s} E V_{\lambda_{i}}$ in (14), and they are $m$ linearly independent extension vectors.

In the following, we discuss the general solutions of the matrix equation (8) by using the solutions of the characteristic equation (9).

Theorem 11. If $\Lambda_{1}$ and $\Lambda_{2}$ are two diverse solutions of the characteristic equation (9), which satisfy $\Lambda_{1} \Lambda_{2}=\Lambda_{2} \Lambda_{1}$, and $\Lambda_{1}-\Lambda_{2}$ is invertible, then

$$
\begin{aligned}
X\left(t_{n}\right)= & \left(\sum_{k=1}^{n} \Lambda_{1}^{k-1} \Lambda_{2}^{n-k}\right) X\left(t_{1}\right) \\
& -\left(\sum_{k=1}^{n-1} \Lambda_{1}^{n-k} \Lambda_{2}^{k}\right) X\left(t_{0}\right)
\end{aligned}
$$

is the general solution of the matrix equation (8).

Proof. Since $\Lambda_{1}$ and $\Lambda_{2}$ are two solutions of (9), then

$$
\begin{aligned}
& \Lambda_{1}^{2}-B \Lambda_{1}-A=0, \\
& \Lambda_{2}^{2}-B \Lambda_{2}-A=0,
\end{aligned}
$$

which deduce

$$
\left(\Lambda_{1}+\Lambda_{2}\right)\left(\Lambda_{1}-\Lambda_{2}\right)=B\left(\Lambda_{1}-\Lambda_{2}\right)
$$

Hence, by the invertibility of $\Lambda_{1}-\Lambda_{2}$, we have

$$
\begin{gathered}
B=\Lambda_{1}+\Lambda_{2}, \quad-A=\Lambda_{1} \Lambda_{2}, \\
\left(\Lambda_{1}-\Lambda_{2}\right)^{2}=B^{2}+4 A .
\end{gathered}
$$

Let $C=\Lambda_{1}-\Lambda_{2}$. It follows from $\Lambda_{1} \Lambda_{2}=\Lambda_{2} \Lambda_{1}$ that

$$
A B=B A, \quad A C=C A, \quad B C=C B .
$$

Replacing $B$ and $A$ with $(1 / 2)(B+C)+(1 / 2)(B-C)$ and $-(1 / 4)(B+C)(B-C)$ in matrix equation $(8)$, we obtain

$$
\begin{aligned}
2 x^{\sigma^{2}}(t)-(B-C) X^{\sigma}(t)= & \frac{1}{2}(B+C) \\
& \times\left[2 X^{\sigma}(t)-(B-C) X(t)\right] .
\end{aligned}
$$

Let

$$
Y(t)=2 X^{\sigma}(t)-(B-C) X(t) .
$$

Then (20) can be rewritten as

$$
Y^{\sigma}(t)=\frac{1}{2}(B+C) Y(t)
$$

Hence, the general solution of (21) is as follows:

$$
Y\left(t_{n}\right)=\frac{1}{2^{n}}(B+C)^{n} Y\left(t_{0}\right),
$$

where $Y\left(t_{0}\right)=2 X\left(t_{1}\right)-(B-C) X\left(t_{0}\right)$. Putting $Y\left(t_{n}\right)$ into (21), we can get the general solution of (8):

$$
\begin{aligned}
x\left(t_{n}\right)= & \frac{1}{2^{n-1}}(B-C)^{n-1} X\left(t_{1}\right) \\
& +\sum_{k=1}^{n-1} \frac{1}{2^{k}}(B-C)^{k-1} Y\left(t_{n-k}\right) \\
= & -\frac{1}{2^{n}} \sum_{k=1}^{n-1}(B+C)^{n-k}(B-C)^{k} X\left(t_{0}\right) \\
& +\frac{1}{2^{n-1}} \sum_{k=1}^{n}(B+C)^{k-1}(B-C)^{n-k} X\left(t_{1}\right) \\
= & \left(\sum_{k=1}^{n} \Lambda_{1}^{k-1} \Lambda_{2}^{n-k}\right) X\left(t_{1}\right) \\
& -\left(\sum_{k=1}^{n-1} \Lambda_{1}^{n-k} \Lambda_{2}^{k}\right) X\left(t_{0}\right) .
\end{aligned}
$$

Theorem 12. If $\Lambda_{1}$ and $\Lambda_{2}$ are two diverse solutions of the characteristic equation (9) and $\Lambda_{1}-\Lambda_{2}$ is invertible, then

$$
X\left(t_{n}\right)=\Lambda_{1}^{n} C_{1}+\Lambda_{2}^{n} C_{2},
$$

where $C_{1}=\left(\Lambda_{2}-\Lambda_{1}\right)^{-1}\left(\Lambda_{2} X\left(t_{0}\right)-X\left(t_{1}\right)\right)$ and $C_{2}=\left(\Lambda_{1}-\right.$ $\left.\Lambda_{2}\right)^{-1}\left(\Lambda_{1} X\left(t_{0}\right)-X\left(t_{1}\right)\right)$, is the general solution of the matrix equation (8).

Proof. Firstly, we prove (25) is a solution of (8). Putting (25) into (8), we have

$$
\begin{aligned}
\left(\Lambda_{1}^{n+2} C_{1}\right. & \left.+\Lambda_{2}^{n+2} C_{2}\right)-B\left(\Lambda_{1}^{n+1} C_{1}+\Lambda_{2}^{n+1} C_{2}\right) \\
& -A\left(\Lambda_{1}^{n} C_{1}+\Lambda_{2}^{n} C_{2}\right) \\
= & \left(\Lambda_{1}^{2}-B \Lambda_{1}-A\right) \Lambda_{1}^{n} C_{1}+\left(\Lambda_{2}^{2}-B \Lambda_{2}-A\right) \\
& \times \Lambda_{2}^{n} C_{2}=0 .
\end{aligned}
$$


By using $X\left(t_{0}\right)=C_{1}+C_{2}, X\left(t_{1}\right)=\Lambda_{1} C_{1}+\Lambda_{2} C_{2}$, and $\Lambda_{1}-\Lambda_{2}$ is invertible, then we get

$$
\begin{aligned}
& C_{1}=\left(\Lambda_{2}-\Lambda_{1}\right)^{-1}\left(\Lambda_{2} X\left(t_{0}\right)-X\left(t_{1}\right)\right), \\
& C_{2}=\left(\Lambda_{1}-\Lambda_{2}\right)^{-1}\left(\Lambda_{1} X\left(t_{0}\right)-X\left(t_{1}\right)\right) .
\end{aligned}
$$

Next, we will prove all the solutions of (8) can be written into the form of (25).

Obviously, by $C_{1}=\left(\Lambda_{2}-\Lambda_{1}\right)^{-1}\left(\Lambda_{2} X\left(t_{0}\right)-X\left(t_{1}\right)\right)$ and $C_{2}=\left(\Lambda_{1}-\Lambda_{2}\right)^{-1}\left(\Lambda_{1} X\left(t_{0}\right)-X\left(t_{1}\right)\right)$, we have $X\left(t_{0}\right)=C_{1}+C_{2}$, $X\left(t_{1}\right) \Lambda_{1} C_{1}+\Lambda_{2} C_{2}$; then $X\left(t_{0}\right)$ and $X\left(t_{1}\right)$ are in the form of (25). Assume the solution of (8) can be written in the form of (25) for $n \leq k$; that is,

$$
X\left(t_{k}\right)=\Lambda_{1}^{k} C_{1}+\Lambda_{2}^{k} C_{2} \text { for } n \leq k .
$$

Then, it follows from (8) and $\Lambda_{1}, \Lambda_{2}$ being solutions of (9) that

$$
\begin{aligned}
x\left(t_{k+3}\right) & =X^{\sigma^{2}}\left(t_{k+1}\right)=B X^{\sigma}\left(t_{k+1}\right)+A X\left(t_{k+1}\right) \\
& =B X\left(t_{k+2}\right)+A X\left(t_{k+1}\right) \\
& =B\left(\Lambda_{1}^{k+2} C_{1}+\Lambda_{2}^{k+2} C_{2}\right)+A\left(\Lambda_{1}^{k+1} C_{1}+\Lambda_{2}^{k+1} C_{2}\right) \\
& =\left(B \Lambda_{1}+A\right) \Lambda_{1}^{k+1} C_{1}+\left(B \Lambda_{2}+A\right) \Lambda_{2}^{k+1} C_{2} \\
& =\Lambda_{1}^{2} \Lambda_{1}^{k+1} C_{1}+\Lambda_{2}^{2} \Lambda_{2}^{k+1} C_{2} \\
& =\Lambda_{1}^{k+3} C_{1}+\Lambda_{2}^{k+3} C_{2} .
\end{aligned}
$$

By using the method of mathematical induction, we have all the solutions of (8) can be written in the form of (25).

\section{Acknowledgments}

This research was supported by the NNSF of China (Grant nos. 11071143 and 11101241), the NNSF of Shandong Province (Grant nos. ZR2009AL003, ZR2010AL016, and ZR2011AL007), and the Scientific Research and Development Project of Shandong Provincial Education Department (J11LA01).

\section{References}

[1] S. Hilger, Ein Ma ßkettenkalkül mit Anwendung auf Zentrumsmannigfaltigkeiten [Ph.D. thesis], Universität Würzburg, 1988.

[2] R. P. Agarwal, M. Bohner, and P. J. Y. Wong, "Sturm-Liouville eigenvalue problems on time scales," Applied Mathematics and Computation, vol. 99, no. 2-3, pp. 153-166, 1999.

[3] R. P. Agarwal and M. Bohner, "Basic calculus on time scales and some of its applications," Results in Mathematics, vol. 35, no. 1-2, pp. 3-22, 1999.

[4] M. Bohner and A. Peterson, Dynamic Equations on Time Scales: An Introduction with Applications, Birkhäuser, Boston, Mass, USA, 2001.

[5] M. Bohner and A. Peterson, Advances in Dynamic Equations on Time Scales, Birkhäuser, Boston, Mass, USA, 2003.
[6] R. P. Agarwal and M. Bohner, "Quadratic functionals for second order matrix equations on time scales," Nonlinear Analysis. Theory, Methods \& Applications, vol. 33, no. 7, pp. 675-692, 1998.

[7] L. Erbe and A. Peterson, "Oscillation criteria for secondorder matrix dynamic equations on a time scale," Journal of Computational and Applied Mathematics, vol. 141, no. 1-2, pp. 169-185, 2002.

[8] M. A. Barkatou, "Rational solutions of matrix difference equations: the problem of equivalence and factorization," in Proceedings of the International Symposium on Symbolic and Algebraic Computation, vol. 99, pp. 277-282, 1999.

[9] G. Freiling and A. Hochhaus, "Properties of the solutions of rational matrix difference equations," Computers \& Mathematics with Applications, vol. 45, no. 6-9, pp. 1137-1154, 2003.

[10] J. Xu and B. Zhang, "The solutions of second order homogenous matrix difference equations," College Mathematics, vol. 20, pp. 96-101, 2004.

[11] Y. Wu and D. Zhou, "The particular solutions to one kind of second order matrix ordinary differential equation," Journal of Foshan University, vol. 29, pp. 14-19, 2011.

[12] J. Huang and J. Chen, "The diagonalizable solution of the quadratic matrix equation $A X^{2}+B X+C=0$," Mathematics in Practice and Theory, vol. 37, pp. 153-156, 2007.

[13] J. Huang and H. Huang, "The solutions of second order linear matrix difference equations and its asymptotic stability," Mathematics in Practice and Theory, vol. 39, pp. 250-254, 2009. 


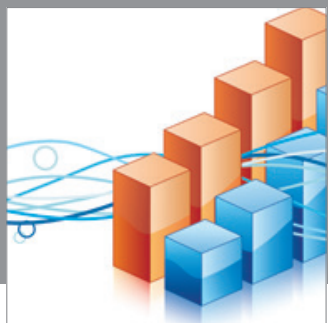

Advances in

Operations Research

mansans

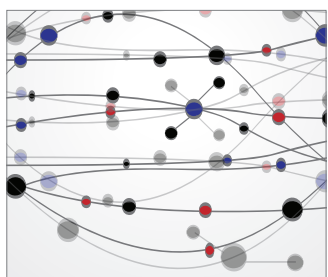

The Scientific World Journal
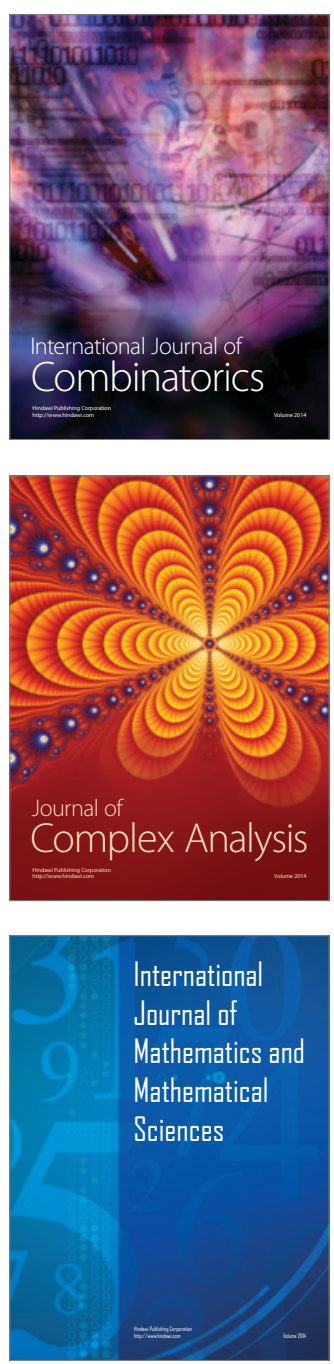
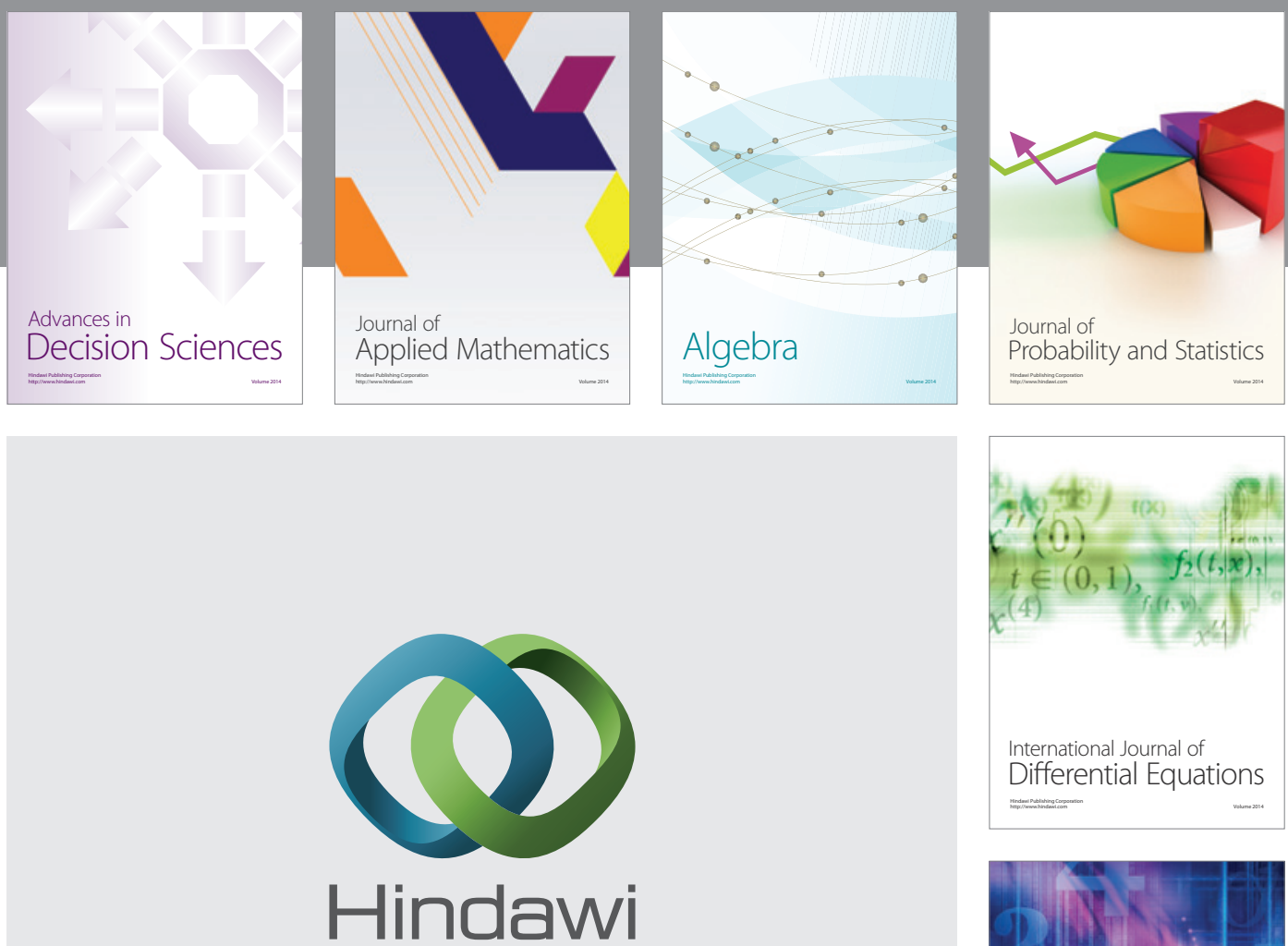

Submit your manuscripts at http://www.hindawi.com
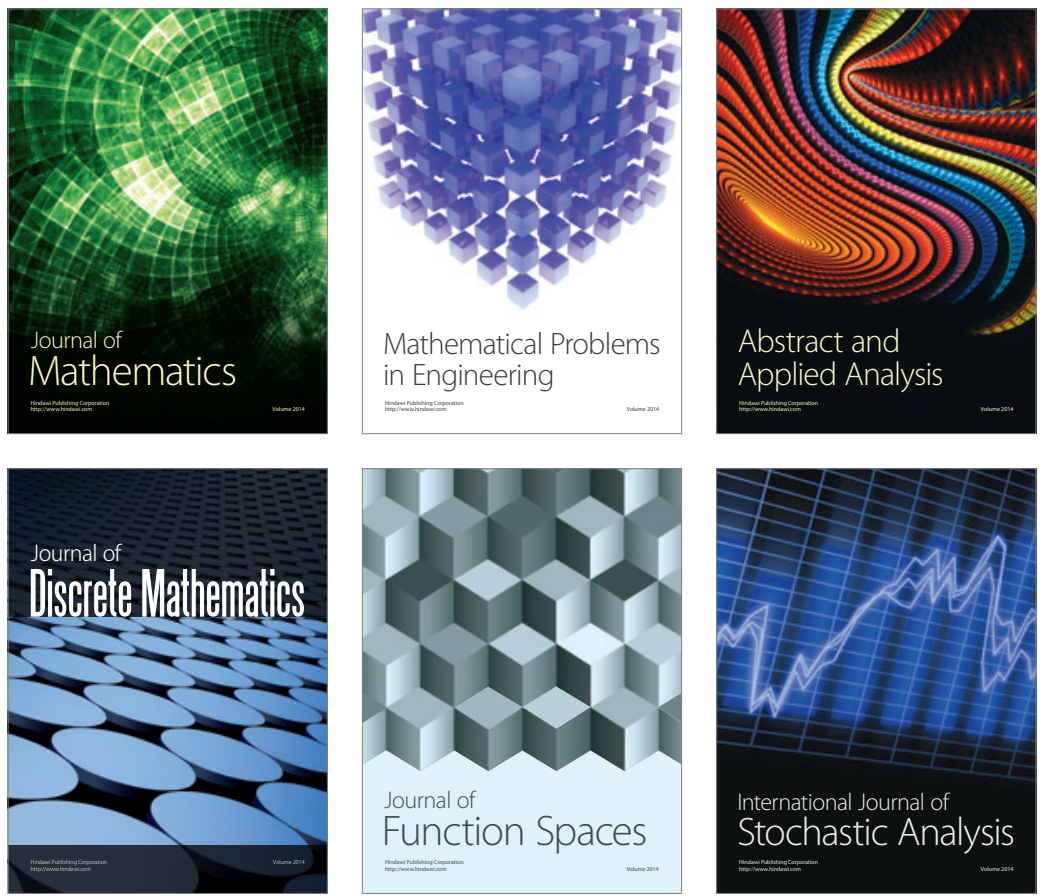

Journal of

Function Spaces

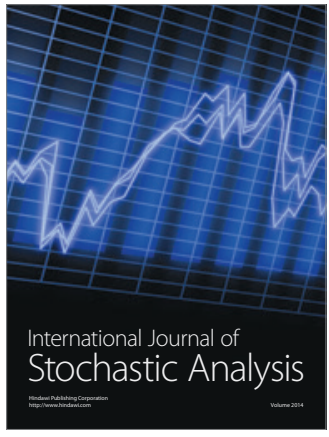

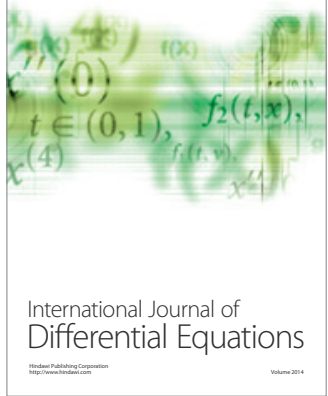
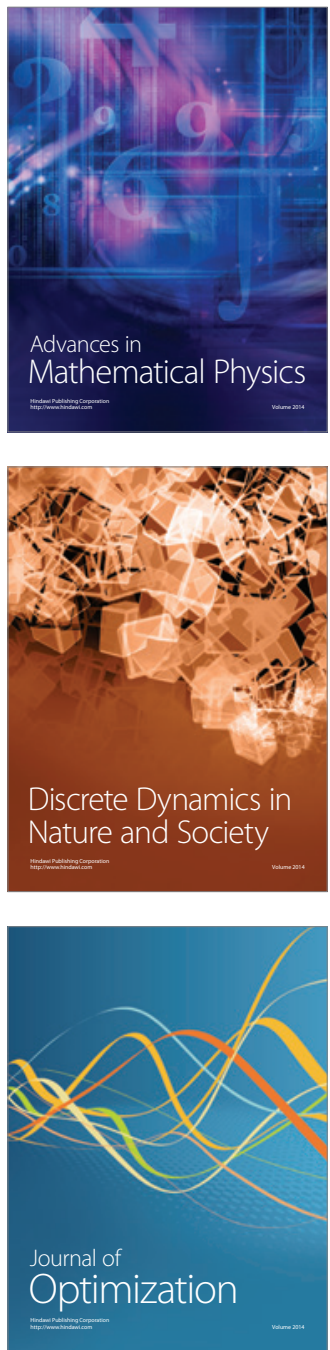